\title{
Self-cooling of a micro-mirror by radiation pressure
}

\author{
S. Gigan ${ }^{1,2}$, H. R. Böhm ${ }^{1,2}$, M. Paternostro ${ }^{2, \dagger}$, F. Blaser ${ }^{1,2}$, G. Langer $^{3}$, J. \\ B. Hertzberg ${ }^{4,5}$, K. Schwab ${ }^{4, \ddagger}$, D. Bäuerle ${ }^{3}$, M. Aspelmeyer ${ }^{1,2 *}$, A. Zeilinger ${ }^{1,2}$ \\ 1 Physics Faculty, Institute for Experimental Physics, \\ University of Vienna, Boltzmanngasse 5, \\ A-1909 Vienna, Austria \\ 2 Institute for Quantum Optics and Quantum Information (IQOQI), \\ Austrian Academy of Sciences, \\ Boltzmanngasse 3, A-1090 Vienna, Austria \\ 3 Institute for Applied Physics, \\ Johannes-Kepler-University Linz, \\ Altenbergerstr. 69, A-4040 Linz, Austria \\ 4 Laboratory for Physical Sciences, \\ University of Maryland, College Park, MD 20740 USA \\ 5 Department of Physics, University of Maryland, \\ College Park, MD 20740, USA \\ $\dagger$ permanent address: School of Mathematics and Physics, \\ Queen's University Belfast, UK \\ ¥ present address: Cornell University, USA
}

(Dated: February 1, 2008)

\begin{abstract}
We demonstrate passive feedback cooling of a mechanical resonator based on radiation pressure forces and assisted by photothermal forces in a high-finesse optical cavity. The resonator is a free-standing high-reflectance micro-mirror (of mass $m \approx 400 \mathrm{ng}$ and mechanical quality factor $Q \approx 10^{4}$ ) that is used as back-mirror in a detuned Fabry-Perot cavity of optical finesse $F \approx 500$. We observe an increased damping in the dynamics of the mechanical oscillator by a factor of 30 and a corresponding cooling of the oscillator modes below $10 \mathrm{~K}$ starting from room temperature. This effect is an important ingredient for recently proposed schemes to prepare quantum entanglement of macroscopic mechanical oscillators.
\end{abstract}

\section{INTRODUCTION}

Cooling of mechanical resonators is currently a hot topic in many fields of physics including ultra-high precision measurements [1] detection of gravitational waves [2, 3] and the study of the transition between classical and quantum behavior of a mechanical system [4, [5, $[6]$. Here, we report the first observation of self-cooling of a micro-mirror by radiation pressure inside a high-finesse optical cavity. In essence, changes in intensity in a detuned cavity, as caused by the thermal vibration of the mirror, provide the mechanism for entropy flow from the mirror's oscillatory motion to the low-entropy cavity field [2]. The crucial coupling between radiation and mechanical motion was made possible by producing free-standing micro-mirrors of low mass $(\mathrm{m} \approx 400 \mathrm{ng})$, high reflectance $(>99,6 \%)$ and high mechanical quality $\left(Q \approx 10^{4}\right)$. We observe cooling of the mechanical oscillator by a factor of more than 30 , i.e. from room temperature to below $10 \mathrm{~K}$. In addition to purely photothermal effects [7] we identify radiation-pressure as a relevant mechanism participating to the cooling. In contrast to earlier experiments, our technique does not need any active feedback [8, 9, 10]. Our results suggest that it should be possible to reach very low temperatures. We expect that improvements of our method will allow for pure radiation pressure cooling, with cooling ratios beyond 1.000, and thus possibly enable cooling all the way down to the quantum mechanical ground state of the micro-mirror.

Radiation pressure forces inside optical cavities are known to pose an ultimate limit on the sensitivity of interferometric measurements [11, 12]. However, less known, radiation pressure can also be used for the opposite, namely to counteract the dynamics of a cavity mirror via dynamical back action [2, 7, 13]. In a recent experiment Metzger and Karrai [7] presented a passive cooling mechanism for a micro-mechanical oscillator based on bolometric back action. Even though this scheme has intrinsically limited cooling capability since it ultimately relies on heating by absorption, it may allow for a quantitatively significant reduction of the oscillator's thermal motion. A more powerful scheme is provided by the use of radiation pressure as a feedback force [2]. In this case, optical absorption does not impose a fundamental limit. The difficulty in utilizing radiation pressure for this cooling purpose is that it requires stable

* Corresponding author: markus.aspelmeyer@quantum.at 
control of the detuning of a high-finesse cavity, strong optomechanical coupling and a low mass of at least one cavity mirror, hence nano- or micro-mechanical systems of high optical and mechanical quality (characterized by the cavity finesse $\mathrm{F}$ and the mechanical quality factor $\mathrm{Q}$ ). Although cavity-induced radiation-pressure effects have already been used to modify elastic properties of mirrors [7, 14, 15, 16] and to enforce mechanical instabilities [14, 17, 18, 19, 20], none of the previous experiments was able to combine these strict requirements. We have overcome this limitation by developing a method to produce free-standing micro-mirrors of low mass $(Q \approx 400 \mathrm{ng})$ high reflectance $(>99.6 \%)$ and high mechanical quality $\left(Q \approx 10^{4}\right)$. Using such micro-mirrors in a detuned optical cavity allows us to observe for the first time self-cooling in a regime where, although photothermal effects are still present, radiation pressure significantly participates in the self-cooling process.

\section{IDEA OF RADIATION-PRESSURE COOLING}

Radiation pressure forces in an optical cavity arise due to the momentum transfer of photons reflected from the mirror surface. For certain cavity detuning, i.e. if the cavity angular frequency $\omega_{c}$ is off resonance with the frequency $\omega_{l}$ of the pump laser, the radiation pressure is highly sensitive to small displacements of the cavity mirror. This is a consequence of the fact that the energy stored in a cavity field varies strongly with detuning. As a consequence, the dynamics of an oscillating mirror inside a detuned cavity is modified by a mechanical rigidity that depends on the detuning. For a high-finesse cavity, the radiation-pressure induced back action can act on the mirror motion in a way to induce low noise damping. This is the general concept of dynamical back action which has first been introduced by Braginsky [13]. A simple classical description of the dynamics of the mirror shows that both the resonance frequency $\omega_{M}$ and the natural damping rate $\gamma$ of the mirror motion are modified by radiation pressure to $\omega_{\text {eff }}$ and $\gamma_{\text {eff }}$, respectively [2, 7]. In particular, within the classical framework, the modified damping rate follows

$$
\gamma_{\mathrm{eff}}=\gamma+\frac{\beta(\Delta)}{2 m} \frac{2 \kappa}{(2 \kappa)^{2}+\omega_{M}^{2}}
$$

with the cavity decay rate $\kappa=\pi c / 2 F L$, the cavity finesse $F$, the cavity length $L$ and the vacuum speed of light $c$. Optimum damping is achieved when $1 / \kappa$ is of the order of $\omega_{M}$, which for $\omega_{M}$ in the MHz range requires a high finesse cavity. Equation (11) depends on $\beta(\Delta)$, the spatial gradient of the radiation force evaluated at a (spatial) detuning $\Delta_{x}=L \Delta / \omega_{l}$. Here, $\Delta$ is the effective detuning between cavity and laser frequency, including the effect of radiation pressure [21]. The contribution $\beta$, induced by radiation pressure, can be positive or negative depending on the sign of $\Delta$. It is straightforward to show that $\beta(\Delta)$ is negative for $\Delta<0$, corresponding to $\gamma_{\mathrm{eff}}<\gamma$. In this regime, the system can enter into instability. The focus of this work is the investigation of the opposite regime $(\beta(\Delta)>0)$ in which $\gamma_{\text {eff }}>\gamma$. This low-noise damping results in a reduction of the mirror temperature and hence self-cooling is achieved. The previous self-cooling experiments based on bolometric forces [7] were operated in the regime of negative detuning where radiation pressure counteracts the cooling.

To observe the self-cooling effect a read-out scheme of the mirror motion is required. To do that it turns out that it is sufficient to measure the statistical properties of the optical field that leaks out of the cavity. In a way, the output cavity field represents a "blank paper" on which the dynamics of the mirror can be written. It is possible to briefly sketch the main idea of our self-cooling read-out process by exploiting a simple (but for our purposes sufficient) semiclassical picture. A full quantum mechanical framework, which generalizes the classical picture for self cooling proposed so far in the literature [2, 10], is presented elsewhere [22]. Not only is this (more general) approach in agreement with the classical picture taken into account by Eq. 1 but it also paves the way toward the rigorous study of the limitations imposed to self cooling by the influences of quantum noise [22]. The total energy of a cavity consisting of a fixed mirror and a movable mirror driven by an input laser field of power $\mathrm{P}$ is given by [21, 23]

$$
E=\hbar\left(\omega_{c}-\omega_{l}\right)\left(X^{2}+Y^{2}\right)-\hbar \frac{\omega_{c}}{2 L}\left(X^{2}+Y^{2}-1\right) q+\frac{1}{2}\left(\frac{p^{2}}{m}+m \omega_{M}^{2} q^{2}\right)+\sqrt{2} \hbar \mathcal{E} Y
$$

where $X$ and $Y$ are the quadratures of the cavity field, $p$ and $q$ are the momentum and position quadratures of the oscillating mirror, and $\mathcal{E}=\sqrt{2 \kappa P / \hbar \omega_{l}}$ is the coupling rate between the cavity and the input laser field. If the time-scale set by the cavity decay rate is the shortest in the dynamics of the system, i.e. $\kappa \gg \omega_{M}$, the cavity field follows the mirror motion adiabatically. As a consequence, the fluctuations $\delta Y_{\text {out }}$ of the field leaking out of the cavity are directly related to the fluctuations of the mirror's position quadrature as $\delta Y(t)=\mathcal{A}(\Delta, \kappa, \mathcal{E}) \delta q(t)$ [22, 24], where we have neglected any noise in the system. For the parameter regime of our experiment the signal-to-noise ratio of the contribution given by the mirror's spectrum is as large as $10^{7}$. The dynamics of the output field quadrature is 
thus entirely determined by the mirror motion via the function $(\Delta, \kappa, \mathcal{E})$. Therefore, a phase-sensitive measurement of the output field quadrature $\delta Y_{\text {out }}$ is capable of "monitoring" the full mirror dynamics. It is particularly interesting to measure the power spectrum, since $S_{Y_{\text {out }}}=\int d t^{\prime} e^{i \omega t^{\prime}}\left\langle\delta Y_{\text {out }}(t) \delta Y_{\text {out }}\left(t+t^{\prime}\right)\right\rangle=\mathcal{T}(\Delta) S_{q}$, where $S_{q}$ is the spectrum of the mirror motion. In other words, the quadrature power spectrum of the mirror motion $S_{q}$ and of the output cavity field $S_{Y_{\text {out }}}$ are directly related via a transfer function $\mathcal{T}(\Delta)$. This correspondence is at the basis of our readoutscheme. Note that the full transfer function has to take into account the sensitivity of the specific detection scheme used. This detection strategy allows us to infer the effective temperature of the mirror Brownian motion through the study of its displacement power-spectrum, i.e. its frequency-dependent mean square displacement. The power spectrum follows a Lorentzian distribution centered around $\widetilde{\omega_{0}}=\sqrt{\omega_{\text {eff }}^{2}-2 \gamma_{\text {eff }}^{2}}$ with a full width at half maximum (FWHM) $w_{\mathrm{FWHM}} \approx 2 \gamma_{\mathrm{eff}}$ (for $\omega_{0}^{2} \gg \gamma_{\text {eff }}^{2}$ ), thus proportional to the introduced damping. The area of the power spectrum, $\left\langle x^{2}\right\rangle=\int_{-\infty}^{+\infty} d \omega S_{q}$, is proportional to the mean energy $\langle E\rangle$ of the vibrational mode and hence, via the equipartition law, to the effective temperature of the mirror, since $\langle E\rangle=m \omega_{M}^{2}\left\langle x^{2}\right\rangle=k_{B} T_{\text {eff }}$. The relative change in area underneath the power spectrum is therefore a direct measure for the change in effective temperature.

\section{EXPERIMENTAL RESULTS AND DISCUSSION}

The system under investigation is a doubly clamped cantilever used as the end mirror of a linear optical cavity driven by an ultra stable Nd:YAG laser (see figure 1). The input mirror of the cavity is attached to a piezo electric transducer which is fed by a control loop allowing us to lock the precise length of the cavity either at resonance or detuned (off resonance) with respect to the laser frequency. The error-signal input to the control loop is obtained via the Pound-Drever-Hall (PDH) technique 25]. It has been shown 24] that the PDH error signal is proportional to the phase quadrature of the output field $Y_{\text {out }}$ and hence to the mirror motion (see above). An intuitive way to view it is that the error signal is proportional to the variation of the cavity length. Above the cut-off frequency of our control loop, the fluctuations in the error signal are therefore directly related to the thermal noise of the cantilever (the input mirror is assumed to be fixed).

We measured the PDH power spectrum for different input powers and cavity detunings. The detuning was achieved by adding an offset to the error signal. With this method, the mechanical damping can be directly measured by determining the FWHM of the resonance peak of the observed mechanical mode. To obtain the effective temperature of the mode one has to calculate the area underneath the resonance peak and to account for the sensitivity of the error signal. This is done by normalizing the measured mirror amplitudes by the gradient of the PDH signal. The results are summarized in Figures 2,3 and 4 .

Figure 2 shows the noise spectrum of the oscillator for two different detunings at $2 \mathrm{~mW}$ input laser power. The width of the peak increases and the area of the peak decreases, indicative for both overdamping and cooling of the mechanical mode. This behavior is in full agreement with the theoretical model presented above. We investigate the specific variation of both mechanical damping and of self-cooling with detuning for different input laser powers of $1 \mathrm{~mW}$ and $2 \mathrm{~mW}$, respectively (Figures 3 and 4). Figure 3 shows the change in width of the mechanical mode. For positive detunings, the peak is broadened from a natural width of $32 \mathrm{~Hz}$ to well above $800 \mathrm{~Hz}$ corresponding to an extra damping of the mode. At large detuning values the stability of the locking limits the precision of the measurements. For negative detuning (not shown), we observed a narrowing of the peak, associated with an amplification of the mirror motion (i.e. "negative" damping), which rapidly leads to a self-oscillation region. In Figure 4 the same data set is used to obtain the corresponding cooling ratio from the relative change in area of the power spectrum, since the total peak area is a measure of temperature. As expected, the increase in damping is accompanied by a cooling of the mechanical mode. At large detuning, the cooling-effect is slightly enhanced compared to our simple model, which can be due to the reduced the contribution of thermal background of other oscillator modes. The best experimental cooling ratio in our detuning range is above 30. Since our experiment is performed at room temperature, this corresponds to a cooling of the mode from $300 \mathrm{~K}$ to below $10 \mathrm{~K}$ (Fig. 2).

We explicitly compare the experimental results for positive detuning with the theoretical predictions obtained if the effect is due only to radiation pressure. To do that, we have independently evaluated the effective mass participating to the dynamics of the system, which leaves no free parameter for the evaluation of radiation-pressure forces and hence allows a full quantitative treatment. The effective mass can be much smaller than the total mass of the cantilever [19, 27]. ]. For our mirror, an independent assessment both via spatial tomography of the vibrational mode and via a calibrated reference results in a value of $224 \mathrm{ng}$ at the probing point (see Appendix). This results in a theoretically expected cooling less strong than the experimentally observed one. To get a clear, immediate figure of the "strength" of the radiation pressure effect required to replicate the experimental data we assume a fixed effective mass and allow for variation of the input power. . We find that, for an effective mass of 18 (26) ng, a power 2.2 (3.3) times larger 


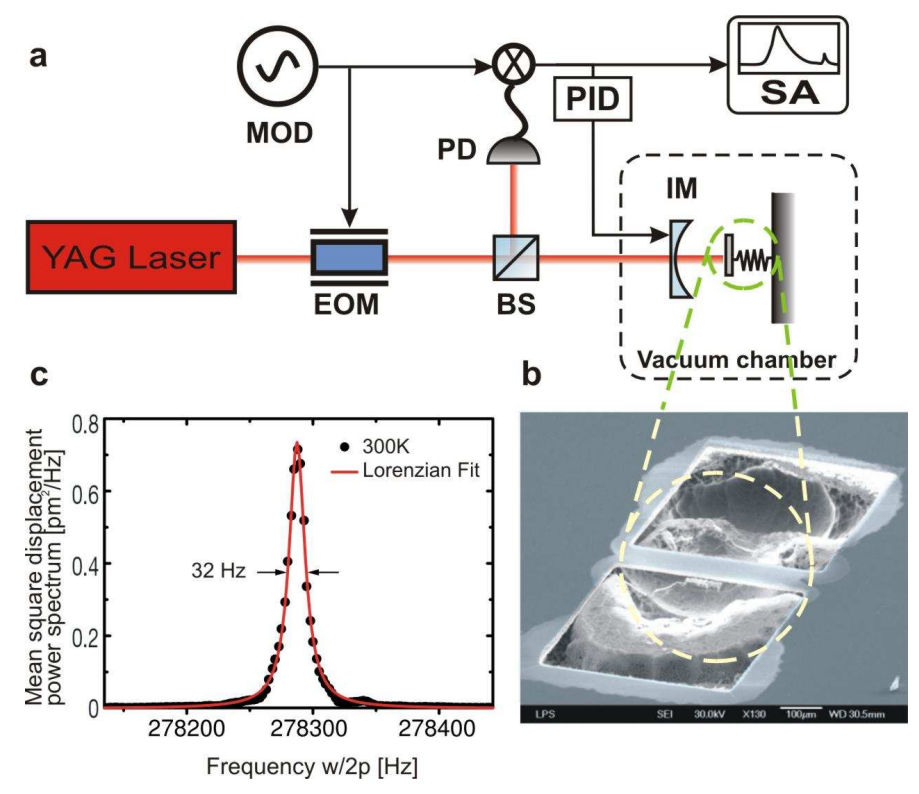

FIG. 1: Sketch of the experimental setup. (a) A cavity is built between the cantilever and a regular concave mirror of 25 mm focal length and $99.3 \%$ reflectivity. The cavity length was slightly shorter than $25 \mathrm{~mm}$ such as to obtain a waist of approximately $20 \mu \mathrm{m}$ at the location of the surface of the cantilever. In this configuration we measured a cavity finesse of 500 . To minimize damping of the mechanical mode due to gas friction, the cavity is placed in a vacuum chamber which is kept at $10^{-5}$ mbar. The cavity is pumped with a Nd:YAG laser at $1064 \mathrm{~nm}$. The beam is phase modulated at $19 \mathrm{MHz}$ (MOD) by a resonant electro-optic modulator (EOM) before it is injected into the cavity via the input mirror (IM). The beam reflected from the cavity is sent via a beam splitter (BS) onto a high-speed PIN photodiode (PD). After amplification of the photocurrent, its AC part is demodulated with the initial modulation frequency to obtain the PDH error signal. This error signal is then used to feed a low-frequency control loop (PID) to stabilize the cavity length via a piezo actuator. In addition, the error signal is fed to a spectrum analyzer (SA) to record the dynamics of the mechanical mode. (b) The cantilever is a doubly clamped free standing Bragg mirror (520 $\mu \mathrm{m}$ long, $120 \mu \mathrm{m}$ wide and $2.4 \mu \mathrm{m}$ thick) that has been fabricated by using UV excimer-laser ablation in combination with a dry-etching process [26]. The reflectivity of the Bragg mirror is $99.6 \%$ at $1064 \mathrm{~nm}$. (c) Power spectrum of the micro-mirror. We have isolated a mechanical mode at $280 \mathrm{kHz}$ with a natural width of $32 \mathrm{~Hz}$, corresponding to $Q \approx 9000$. All measurements presented in this work have been made on this mode.

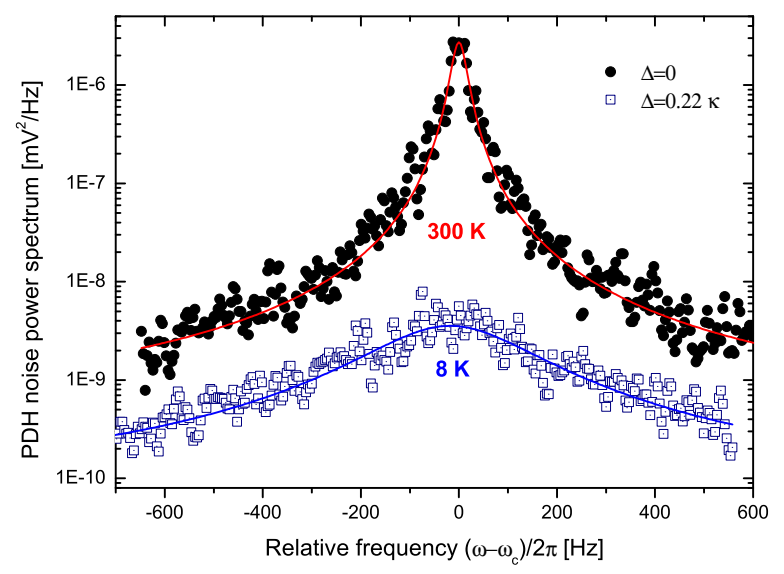

FIG. 2: Power spectrum of the mechanical mode at two different relative detuning levels $\Delta$ of the cavity for an input power of $2 \mathrm{~mW}$. The data is obtained from the PDH power spectrum, which is directly proportional to the displacement power spectrum of the micro-mirror. Experimental points are taken with the spectrum analyzer, averaged over 30 consecutive measurement runs. Solid lines are Lorentzian fits to the data. The areas obtained from the fit correspond to temperatures of $300 \mathrm{~K}$ and 8 $\mathrm{K}$, respectively. 


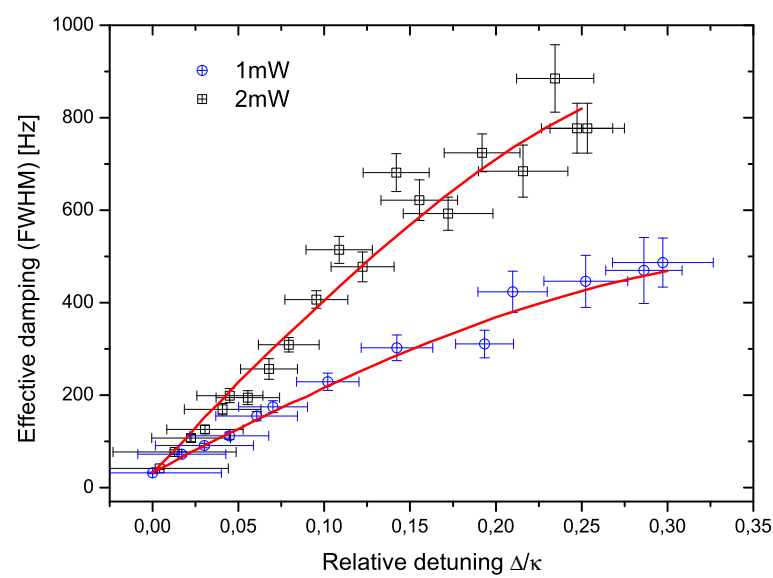

FIG. 3: Radiation-pressure induced damping of mirror dynamics. We show the measured width of the mechanical mode at $278 \mathrm{kHz}$ at different detuning levels of the cavity and for input laser powers of $1 \mathrm{~mW}$ and $2 \mathrm{~mW}$. The data is obtained directly from Lorentzian fits on the measured power spectra of the PDH error signal. Error bars represent absolute errors based on experimental uncertainty. Solid lines represent theoretical predictions of purely radiation-pressure effects for $F=500$, $Q=9000$, an effective mass of $9 \mathrm{ng}$ and input powers of $1 \mathrm{~mW}$ and $2 \mathrm{~mW}$, respectively. The inferred effective mass of $224 \mathrm{ng}$ indicates the presence of an additional damping force of photothermal nature (see text).

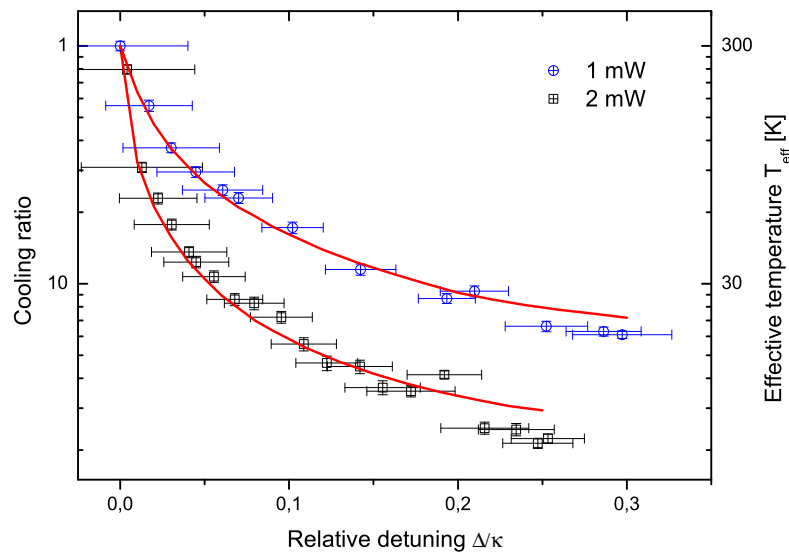

FIG. 4: Self-cooling of the mechanical resonator. We show the cooling ratio on the mechanical mode as a function of detuning and for input laser powers $1 \mathrm{~mW}$ and $2 \mathrm{~mW}$. The data is obtained as normalized area of the measured PDH power spectrum, compensated for the detuning dependent sensitivity of the PDH cavity response. Error bars represent absolute errors based on experimental uncertainty. The self-cooling effect increases for increasing laser power and detuning, in agreement with the theoretical predictions (solid lines). The right axis shows the inferred effective temperature of the mechanical oscillator. Radiation pressure contributes between $30 \%$ and $50 \%$ to the overall cooling, which is assisted by photothermal effects.

than the nominal value used in the experiment is required in order to match the theoretical predictions with both the observed damping and cooling In other words, radiation pressure accounts for at least $30 \%$ of the observed cooling but may be as strong as $50 \%$, i.e. cooling by a factor between 8 and 12 . We attribute the additional cooling in our setup to the presence of photothermal effects. Similar to the bolometric forces reported in 7], differential heating of the outer layers of the dielectric Bragg mirror can result in time-delayed changes of the cavity length eventually introducing a retarded force that can contribute to the self-cooling mechanism. In a thin-layered medium the delayed force induced by photothermal effects can have typical time constants on the order of several tens of ns (see Appendix), fast enough 
to compete with the time scale of radiation pressure effects on the order of $1 /(2 \kappa)$ (approx. $13 \mathrm{~ns}$ in our experiment). The direction of the force depends on the specific material properties of the expanding layers. In our case and in contrast to previous experiments it assists the cooling effect of radiation pressure present for positive detuning.

The experimental data is consistent with radiation-pressure cooling assisted by photothermal effects. Residual heating of the cantilever due to absorption could not be observed (see Appendix). Improvements of the Bragg mirror reflectivity will further reduce and eventually eliminate photothermal contributions to the cooling since it will allow to achieve a higher finesse and to limit the optical absorption. An interesting analogy to understand this cooling mechanism can be found in thermodynamics. If a system (the mirror), initially at thermal equilibrium with a bath at ambient temperature (its environment), is strongly coupled to another bath with a very low temperature (the lownoise laser), its temperature will decrease in order to bring it to equilibrium with both baths. The current technical limitation for observing a lower temperature is the stability of the detuned locking and the base temperature from which the self-cooling starts. For example, with a cavity finesse $F=6000$ and $1 \mathrm{~mW}$ of input optical power we expect a pure radiation pressure cooling ratio of 1500 for a smaller mirror oscillating at $1 \mathrm{MHz}$ with an effective mass of $5 \mathrm{ng}$ and $Q=10^{5}$. Starting from $5 \mathrm{~K}$, one should achieve cooling to $3 \mathrm{mK}$, below the base temperature of a dilution fridge. We are confident that the quantum ground state may be reachable with state-of-the-art optics and microfabrication technique [28].

\section{CONCLUSION}

We have observed self-cooling of a micro-mirror sustained by radiation pressure. The cooling of mechanical oscillators is a key requirement for many open problems of modern physics ranging from the performance of shot-noise limited position measurements [1] to the study of gravitational waves [2, 3] and dynamical multistability in microoptical systems [29]. The possibility of lowering the temperature of an oscillator to its quantum mechanical ground state paves the way to the implementation of quantum state engineering involving macroscopic systems [23, 30, 31], a closer study of the boundary between classical and quantum physics [6] and, ultimately, the observation of nonclassical correlations between macroscopic objects [28]. In the long run it may also provide new means for integrated quantum (mechanical) information processing.

We would like to thank C. Brukner, S. Gröblacher, J. Kofler, T. Jennewein, M. S. Kim, and D. Vitali for discussion. We acknowledge financial support by the Austrian Science Fund (FWF), by the City of Vienna and by the Austrian NANO Initiative (MNA). 


\title{
APPENDIX
}

\section{Mechanical characterization of the vibrational mode}

\author{
CANTILEVER CHARACTERISTICS
}

The doubly clamped cantilever is a free standing Bragg mirror made of several layers of $\mathrm{TiO}_{2} / \mathrm{SiO}_{2}$. The characteristics of the Bragg mirror are summarized in table 1 .

TABLE I: Properties of the Bragg mirror

\begin{tabular}{|c|c|c|c|c|c|}
\hline Material & $\begin{array}{c}\text { Density } \\
\left(\mathrm{kg} / \mathrm{m}^{3}\right)\end{array}$ & $\begin{array}{c}\text { Layer } \\
\text { Thickness }(\mathrm{nm})\end{array}$ & $\begin{array}{c}\text { Number } \\
\text { of layers }\end{array}$ & $\begin{array}{c}\text { Index of } \\
\text { refraction }\end{array}$ & $\begin{array}{c}\text { Bulk thermal } \\
\text { diffusivity } \mathrm{cm}^{2} / \mathrm{s}\end{array}$ \\
\hline $\mathrm{SiO}_{2}$ & 2200 & 183.45 & 8 & 1.45 & 0.086 \\
\hline $\mathrm{TiO}_{2}$ & 4200 & 107.26 & 9 & 2.48 & 0.031 \\
\hline
\end{tabular}

The dimensions of the structure are set by the laser-ablated pattern to $490 \mu \mathrm{m} \times 110 \mu \mathrm{m}$, which corresponds to a total mass of $390 \mathrm{ng}$. However the ends of this structure are not totally undercut and therefore not free to move. This means that the actual cantilever is shorter and the total mass participating in the oscillation is lower.

\section{MODE TOMOGRAPHY}

The mechanical mode has been spatially characterized by scanning the surface of the cantilever and performing point-by-point measurements of the mean square displacement at the position of the optical beam. The measurement has been done at low power (input power $\approx 200 \mu \mathrm{W}$ ) and at zero detuning to avoid spurious radiation-pressure effect. By performing a longitudinal scan (approximately 40 points regularly spaced by $12 \mu \mathrm{m}$ ) along a line close to the center of the bridge, we reconstructed the transverse profile of the first three modes and compared it with theoretical simulations (Fig. 51):

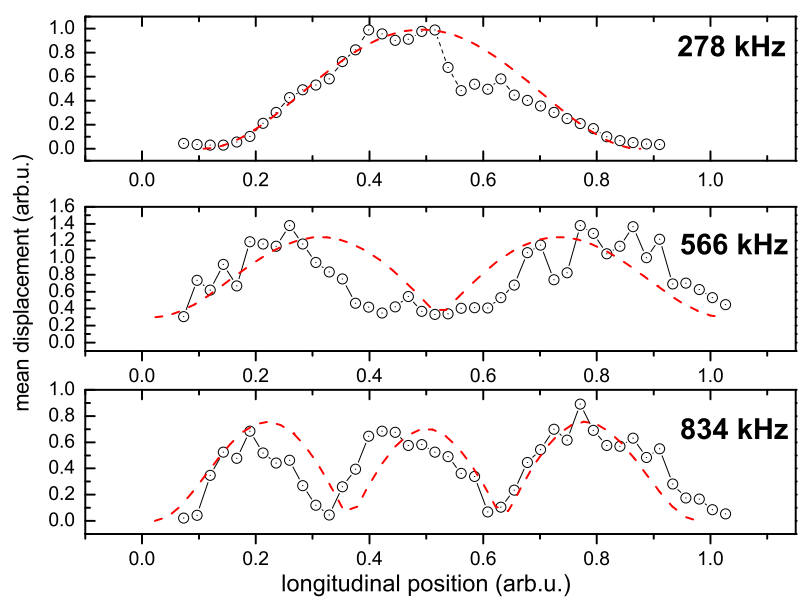

FIG. 5: Mode shape of the first three modes of an ideal doubly-clamped beam: Experimental points (dots) and comparison with the theoretical shape for the first three longitudinal modes of a doubly clamped bridge (red line).

From this analysis, we conclude that the oscillation along the longitudinal direction of the mode at $280 \mathrm{kHz}$ is a fundamental mode. The frequency pattern is consistent with a tension-dominated vibration since higher order mode frequencies are almost harmonics of the fundamental one (i.e. close to the ratio 2:1 and 3:1 respectively).

In order to determine the effective mass relevant for radiation-pressure effects (see below), we have performed a 2D tomography (approximately 15 x 10 points, corresponding to a mesh of roughly $10 \mu \mathrm{m} \times 50 \mu \mathrm{m}$ ) of the observed mechanical mode at $280 \mathrm{kHz}$ (Fig. 6). We see a clear decrease in the amplitude of oscillation when moving laterally 
towards one side of the cantilever. It is important to note that the coating is slightly damaged by the laser ablation close to this side of the structure. This means that approximately $30 \%$ of the cantilever cannot be addressed. From the mode shape we conclude that this part does not participate in the vibration of the mode. This spatial behavior has been modeled by considering a transverse behavior consistent with an additional clamping on one side, as shown in Fig. 6. The agreement in the shape of the mode is evident.
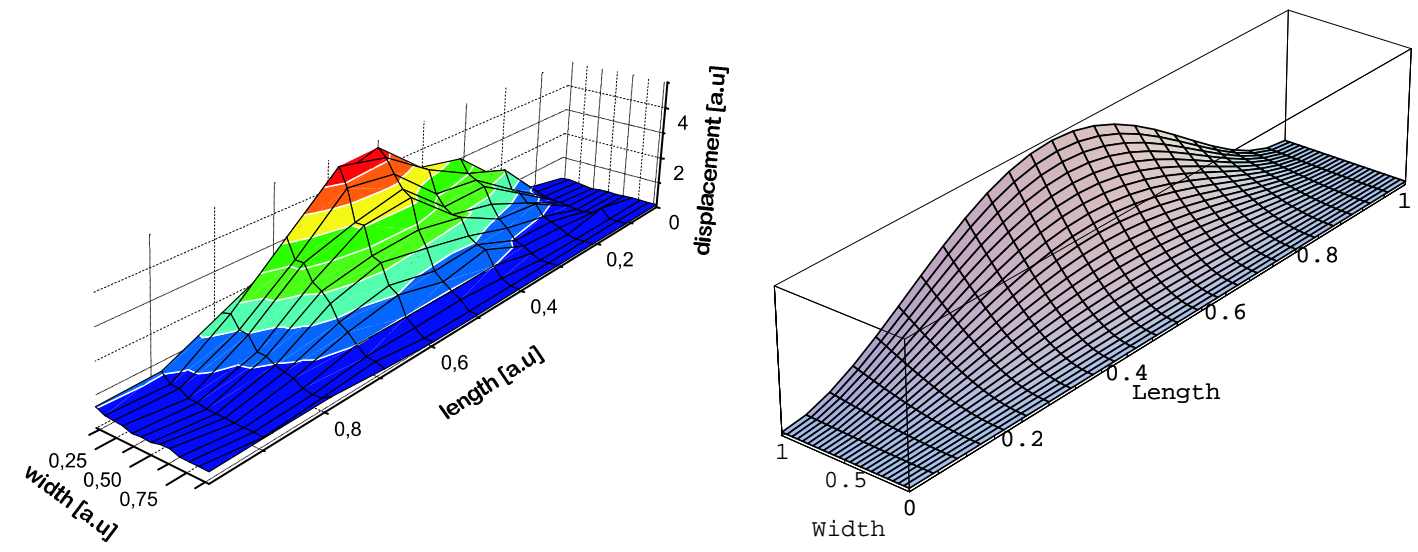

FIG. 6: 2D tomography of the mean displacement of the $280 \mathrm{kHz}$ vibrational mode (measurement (left) and simulation (right) )

The cantilever does not behave as an ideal doubly-clamped cantilever but as if approximately half of it is not resonating at $280 \mathrm{kHz}$, resulting in an apparent clamping on one side.

\section{EFFECTIVE MASS}

It is not possible to simply consider the probed mechanical mode as a point-like oscillator and to associate to it the total mass of the object. One has to take into account the spatial point and extent over which the probing is done, since the amplitude of the vibrations is position-dependent. In other words, the mass which enters in the mechanical susceptibility $x[\Omega]=\chi[\Omega] F[\Omega]$, written as:

$$
\chi[\Omega]=\frac{1}{M_{e f f}\left(\Omega_{M}^{2}-\Omega^{2}-i \frac{\Omega_{M} \Omega}{Q}\right)}
$$

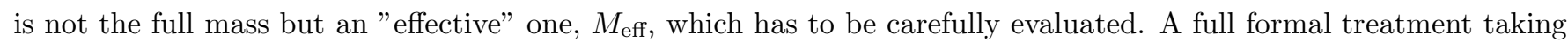
this effect into account has been developed and experimentally demonstrated in [27, 32]. We will explain it briefly, in order to provide an independent evaluation of $M_{\text {eff }}$ in our particular case.

For a point-dependent force $F(r, t)$, acting so to excite a displacement mode of the cantilever normal to its surface, the total work can be written as $W(t)=-<F(r, t), u(r, t)\rangle$, where the bracket stands for a spatial integration over the cantilever's surface. For radiation pressure force from an optical beam with spatial profile $v(r)$ and intensity $I(t)$, the force can be written as:

$$
F_{r a d}=2 \hbar k v^{2}(r) I(t)
$$

where we have normalized the profile $\mathrm{v}$ as $\left\langle v^{2}\right\rangle=1$. The effective susceptibility $\chi_{\text {eff }}$ involves then the effective mass $M_{\text {eff }}$ reading $M_{\text {eff }}=\rho_{s} \frac{<u^{2}>}{\left.<u, v^{2}\right\rangle^{2}}$ with $\rho_{s}$ the surface density. This definition is independent of the normalization of $u(r, t)$. If one considers a point-like probing at position $r_{0}$, the term $<u^{2}>/<u, v^{2}>^{2}$ reduces to $<u^{2}>/ u^{2}\left(r_{0}\right)$, which is the ratio between the mean square displacement at point $r_{0}$ and the square displacement over the surface of the mode. In other words, this formalizes the localization of the mode. This is even clearer when one considers a higher-order mode of oscillation. In this case the mode shape presents nodes (anti-nodes) of oscillation, corresponding to points or regions where the amplitude is zero (maximum). By probing one particular mode on its node, the 
amplitude of the oscillation will be zero, corresponding to an infinite effective mass. On the other hand, if one has to probe at an antinode, the effective susceptibility to the probe is maximally enhanced compared to the ideal point-like oscillator, so that $M_{\text {eff }}$ can be strongly reduced with respect to the total mass.

From the tomography of the mode (Fig. 6), we obtain a lower bound (at the antinode) for the mass magnification ratio $\left\langle u^{2}>/ u^{2}\left(r_{0}\right)\right.$ of $1 / 10$. The estimation of this factor is limited by the coarse-grained tomographic reconstruction of the mode since it depends on the square of the mean displacement. The estimation of the exact mass participating to the oscillations, for the different reasons stated above, is difficult. However, there is clear evidence that a potentially large fraction of the bridge does not contribute to the total mass (reasonably estimated to $\approx 50 \%$ of the mass). Taking the full mass of the bridge as an upper bound for the total mass and 1/10 as a lower bound for the magnification ratio due to the localization of the mode, we obtain in $39 \mathrm{ng}$ a very conservative upper bound for the effective mass, and a realistic evaluation for the effective mass around $20 \mathrm{ng}$.

We have performed an independent evaluation of the effective mass. Assuming room temperature (300 K), we can deduce via the equipartition theorem from the thermal motion of the cantilever the effective mass at the probing point. We send $0.1 \mathrm{~mW}$ of power in the cavity and measure the noise power of the light in a frequency window around $280 \mathrm{kHz}$. By knowing the cavity characteristics and by carefully calibrating the electronic detection scheme, one obtains from the measured intensity modulation the (calibrated) mean square displacement of the mirror and, via the equipartition theorem, the effective mass. Our analysis yields a value of $22 \pm 4 \mathrm{ng}$ at the antinode.

\section{Cavity characteristics}

The input cavity is a massive mirror ( 0.5 " diameter, with radius of curvature $\mathrm{R}=25 \mathrm{~mm}$ ) with a measured reflectivity of $99.3 \pm 0.2 \%$. The reflectivity of the micro-mirror is evaluated to be $99.7 \pm 0.2 \%$ by measuring the cavity finesse outside of the bridge. On the cantilever the finesse is reduced, probably due to scattering and diffraction losses close to the edges of the cantilever. The cavity length is chosen to be close to a semi-concentric configuration (consisting of a length slightly smaller than $25 \mathrm{~mm}$ which characterizes semi-concentricity). In this configuration, the size of the cavity waist is very sensitive to minute changes of the cavity length and can be adjusted to be much smaller than the bridge width, so as to reduce scattering and edge-diffraction. By careful positioning on the cantilever, one can obtain a finesse as large as 500, corresponding to a waist diameter of approximately $20 \mu \mathrm{m}$ and to roughly $0.2 \%$ extra losses.

\section{Assessement of the photothermal contribution}

\section{Manifestation of Heating}

When scanning the length of the cavity over more than a free spectral range (by sending a ramp voltage to the piezo electric transducer on which the massive input mirror is placed), one can record the intensity reflected from the cavity (see Fig. 7 for the reflection peak corresponding to an input power of $2 \mathrm{~mW}$ ) and measure the finesse. This is given by the width of the reflection peak divided by the free spectral range. From Fig. 7 we can see that there is no clear asymmetry in the peak shape, whose presence would be a manifestation of strong heating effects.

It is possible to evaluate the photo-absorption and the consequent heating of the cantilever by comparing the temperature of the mode, proportional to the area underneath a mechanical resonance peak, and the corresponding width, for different input powers. The results are summarized in Fig. 8

By looking at the behavior corresponding to a given input power, we see that width and temperature are inversely related, as expected from the self-cooling mechanism. However, by comparing the curves obtained at different input power, we can also gather information about the heating of the cantilever. If no residual heating takes place, all points will fall on the same line irrespective of the laser power, since cooling is only given by the effective damping in the system. Heating would be revealed by a shift of the data set belonging to a certain laser power as compared to a data set at lower laser power. On the contrary, the experimental curves in Fig. 8 are, within the error bars, superimposed in a way that the described stacking cannot be observed. This implies there is no discernable heating of the cantilever within the range of input powers we have considered. Furthermore, Fig. 8 shows that even if some residual heating is present (which, within the error bar, is not the case), it does not undermine the self-cooling performance in our experiment. 


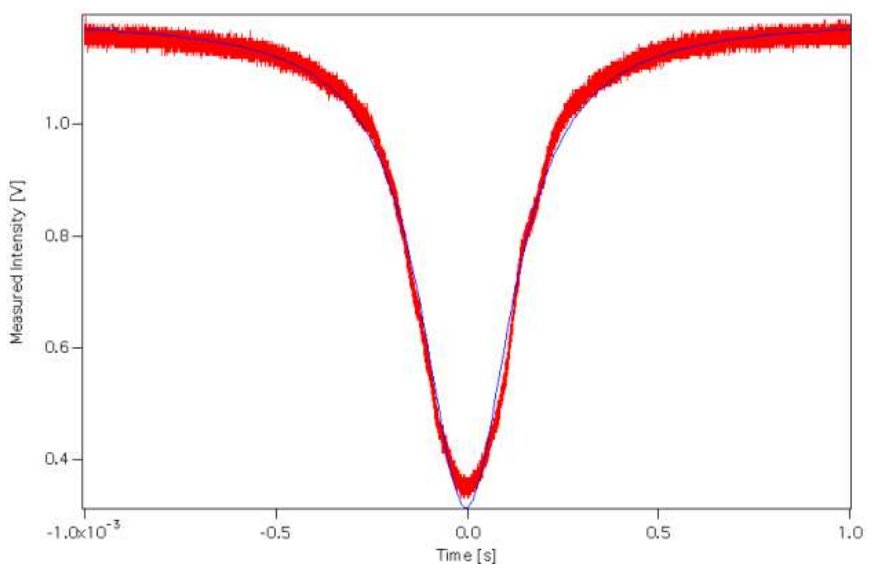

FIG. 7: Experimental length scan of the cavity around a reflection peak for an input power of $2 \mathrm{~mW}$ (red), and Lorentzian fit (blue). ). The cavity is slowly scanned in time. The FWHM of the lorentzian correspond to $\lambda / F \approx 2 n m$

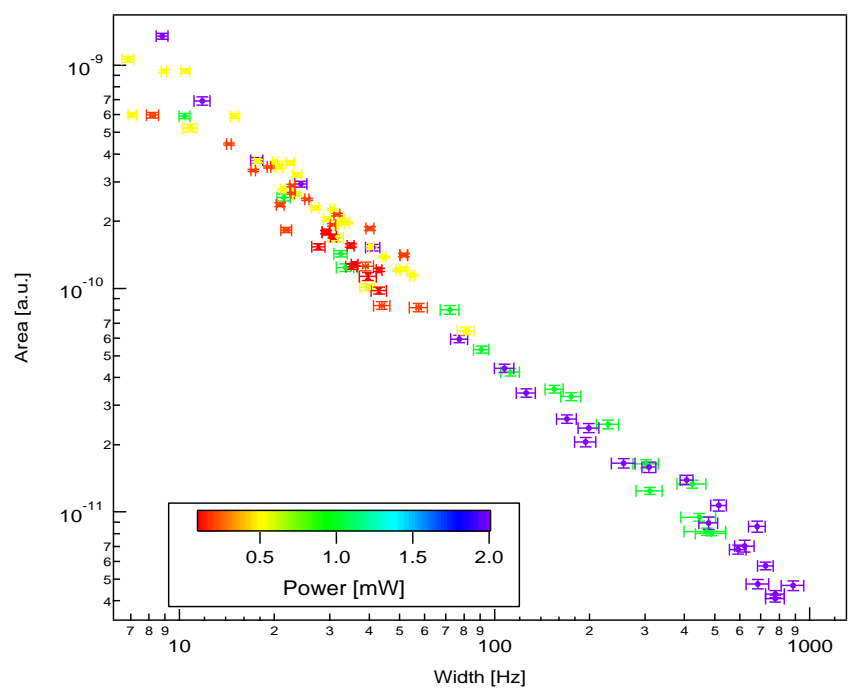

FIG. 8: Area versus width for different input power (see text).

Timescale of the photothermal effects

The mechanical and thermal properties of our free standing layered structures are not easy to assess. Mechanical and thermal properties of thin layered microstructures are not well known and can strongly differ from the those of bulk materials [33]. It is therefore difficult to measure or calculate the exact timescale and strength of the photothermal effects in our experiment. The inferred strength of the photothermal contribution to the cooling of the micromechanical oscillator suggests that the effect is at the same time strong and very fast (i.e. much faster than the period of oscillation of the mode, and in competition with the typical radiation pressure response time $1 / \kappa=13 \mathrm{~ns}$ ). Usually, photothermal processes are typically relatively slow processes. However, we will give a simple and rough evaluation of the thermalisation time, showing that a timescale as fast as the radiation pressure response time, in our conditions, is easily achievable. We can consider that the lateral dimension of the heat-affected zone on the micromirror is roughly the size of the waist of the cavity $(20 \mu \mathrm{m})$. The evanescent wave on the Bragg mirror has a penetration depth which is below 0.4 microns. This means that the heating due to absorption mainly affects the first couple of layers in the structure. Due to different absorption coefficients, these first layers heat up differently over their whole lateral dimension. As a mechanism for thermalisation, we can therefore restrict ourselves to a one-dimensional heat diffusion process between the two first layers of material that is causing the thermalization. The timescale of the fastest photothermal forces should be of the order of this thermalisation time, assuming an initial difference of temperature between the two first layers. Assuming that the usual relation for the heat diffusion length $l_{T}=\zeta \sqrt{D \tau}$ 
holds for such thin layers (where $l_{T}$ is the diffusion length after a time $\tau, \zeta$ is a geometrical factor roughly in the order to of unity, and $D$ is the thermal diffusivity), and assuming the mean bulk values for $D$ (see table I), one gets an estimated thermalisation time for the two layers of typically about $\tau=\left(\frac{1}{\zeta}\right)^{2}\left(\frac{L_{1}^{2}}{D_{1}}+\frac{L_{2}^{2}}{D_{2}}\right) \approx 4$ ns(with $L_{1}$ and $L_{2}$ the thickness of the two layers and $D_{1}$ and $D_{2}$ the thermal diffusivities of the two materials). The square dependence of the thermalisation time on the dimension of the structure allows for a relaxation time range of a few nanoseconds, which determine the contribution of this processes in the cooling mechanism.

\section{REFERENCES}

[1] M. D. LaHaye, O. Buu, B. Camarota, and K. C. Schwab, Science 304, 74 (2004).

[2] V. Braginsky and S. P. Vyatchanin, Physics Letters A 293, 228 (2002).

[3] Ligo, URL http://www.ligo.caltech.edu/

[4] K. C. Schwab and M. L. Roukes, Physics Today (2005).

[5] A. J. Leggett, J. Phys.: Condens. Matter 14, R415 (2002).

[6] W. Marshall, C. Simon, R. Penrose, and D. Bouwmeester, Phys. Rev. Lett. 91, 130401 (2003).

[7] C. Metzger and K. Karrai, Nature 432, 1002 (2004).

[8] J. Mertz and A. Heidmann, J. Opt. Soc. Am. B. 10, 745 (1993).

[9] P. Cohadon, A. Heidmann, and M. Pinard, Phys. Rev. Lett. 83, 3174 (1999).

[10] P. Bushev, D. Rotter, F. Dubin, C. Becher, J. Eschner, R. Blatt, V. Steixner, P. Rabl, and P. Zoller, Phys. Rev. Lett 96, 043003 (2006).

[11] C. Caves, Phys. Rev. Lett. 45, 75 (1980).

[12] V. Braginsky, S. Strigin, and S. P. Vyatchanin, Physics Letters A 287, 331 (2001).

[13] V. Braginsky and F. Khalili, Quantum Measurements (Cambridge University Press, 1995).

[14] R. Tucker, D. Baney, W. Sorin, and C. Flory, IEEE Journal of Selected Topics in Quantum Electronics 8, 88 (2002).

[15] M. Vogel, C. Mooser, K. Karrai, and R. Wartburton, Applied Physics Letters 83, 1337 (2003).

[16] B. Sheard, M. Gray, C. Mow-Lowry, and D. McClelland, Phys. Rev. A 69, 051801 (2004).

[17] A. Dorsel, J. McCullen, P. Meystre, E. Vignes, and H. Walther, Phys. Rev. Lett. 51, 1550 (1983).

[18] H. Rokhsari, T. Kippenberg, T. Carmon, and K. Vahala, Optics Express 13, 5293 (2005).

[19] T. Kippenberg, H. Rokhsari, T. Carmon, A. Scherer, and K. Vahala, Phys. Rev. Lett. 95, 033901 (2005).

[20] T. Carmon, H. Rokhsari, L. Yang, T. Kippenberg, and K. Vahala, Phys. Rev. Lett. 94, 223902 (2005).

[21] V. Giovanetti and D. Vitali, Phys. Rev. A 63, 023812 (2001).

[22] M. Paternostro, S. Gigan, M. S. Kim, F. Blaser, H. R. Böhm, and M. Aspelmeyer, N. J. Phys. 8, 107 (2006).

[23] J. Zhang, K. Peng, and S. L. Braunstein, Phys. Rev. A 68, 013808 (2003).

[24] K. Jacobs, I. Tittonen, H. Wiseman, and S. Schiller, Phys. Rev. A 60, 538 (1999).

[25] E. D. Black, Amer. Jour. of Phys. 69, 79 (2001).

[26] D. Bäuerle, Laser Processing and Chemistry (Springer, 2000), 3rd ed.

[27] M. Pinard, M. Y. Hadjar, and A. Heidmann, Eur. Phys. J. D 7, 107 (1999).

[28] M. Pinard, A. Dantan, D. Vitali, O. Arcizet, T. Briant, and A. Heidmann, Europhys. Lett. 72, 747 (2005).

[29] F. Marquardt, J. Harris, and S. Grivin, eprint arXiv:cond-mat/0502561 (2005).

[30] S. Bose, K. Jacobs, and P. L. Knight, Phys. Rev. A 56, 4175 (1997).

[31] S. Mancini and P. Tombesi, Phys. Rev. A 49, 4055 (1994).

[32] T. Briant, P. Cohadon, A. Heidmann, and M. Pinard, Phys. Rev. A 68 (2003).

[33] S. Lee, D. Cahill, and T. Allen, Phys. Rev. B 52, 253 (1995). 